\title{
Infinitely many planar cubic hypohamiltonian graphs of girth 5
}

\author{
Jan Goedgebeur* \\ DEPARTMENT OF APPLIED MATHEMATICS, COMPUTER SCIENCE \\ AND STATISTICS \\ GHENT UNIVERSITY \\ KRIJGSLAAN 281 - S9, 9000 GHENT, BELGIUM \\ E-mail: jan.goedgebeur@ugent.be \\ Carol T. Zamfirescu* \\ DEPARTMENT OF APPLIED MATHEMATICS, COMPUTER SCIENCE \\ AND STATISTICS \\ GHENT UNIVERSITY \\ KRIJGSLAAN 281 - S9, 9000 GHENT, BELGIUM \\ E-mail: czamfirescu@gmail.com
}

In loving memory of Ella.

\begin{abstract}
A graph $G$ is hypohamiltonian if $G$ is non-hamiltonian and for every vertex $v$ in $G$, the graph $G-v$ is hamiltonian. McKay asked in [J. Graph Theory 85 (2017) 7-11] whether infinitely many planar cubic hypohamiltonian graphs of girth 5 exist. We settle this question affirmatively. (c) John Wiley 8 Sons, Inc.

Keywords: Hamiltonian, hypohamiltonian, planar, cubic, dot product
\end{abstract}

* Both authors are supported by a Postdoctoral Fellowship of the Research Foundation Flanders (FWO).

Journal of Graph Theory Vol. ???, 1-8 ()

(c) John Wiley \& Sons, Inc.

CCC 


\section{JOURNAL OF GRAPH THEORY}

\section{INTRODUCTION}

Every graph in this paper will be undirected, finite, connected, and contain neither loops nor multiple edges, unless stated otherwise. A graph is hamiltonian (traceable) if it contains a cycle (path) visiting every vertex of the graph - such a cycle or path is called hamiltonian. A graph $G$ is hypohamiltonian (hypotraceable) if $G$ itself is non-hamiltonian (non-traceable), but for every vertex $v$ in $G$, the graph $G-v$ is hamiltonian (traceable).

The study of hypohamiltonicity goes back to a paper of Sousselier [15] from the early sixties. The 1993 survey of Holton and Sheehan [8] provides a good overview. For the latest developments we refer the reader to the following articles and the references found therein: the papers $[6,7]$ by the two authors of this work, and articles by Jooyandeh, McKay, Östergård, Pettersson, and the second author [11], McKay [12], and the second author [18]. Ozeki and Vrána [13] recently used hypohamiltonicity to show that there exist infinitely many graphs which are 2-hamiltonian but not 2-edge-hamiltonianconnected, while Wiener [17] makes heavy use of hypohamiltonian graphs in his study of leaf-stable and leaf-critical graphs.

We call a vertex cubic if it has degree 3 , and a graph cubic if all of its vertices are cubic. Consider a graph $G$. Two edges of $G$ are independent if they have no common vertices. The girth of a graph is the length of its shortest cycle.

\section{MAIN RESULT}

In [12] McKay constructed three planar cubic hypohamiltonian graphs of girth 5 and raised the natural question whether infinitely many such graphs exist. We will now prove that this question has a positive answer.

Let $G$ be a graph containing vertices $v, w, x, y$. Following Chvátal [4], the pair of vertices $(v, w)$ is good in $G$ if there exists a hamiltonian path in $G$ with end-vertices $v$ and $w$. Two pairs of vertices $((v, w),(x, y))$ are good in $G$ if there exist two disjoint paths which together span $G$, and which have end-vertices $v$ and $w$, and $x$ and $y$, respectively.

Let $H$ and $H^{\prime}$ be cubic graphs on at least six vertices. Consider $J=H-\{a b, c d\}$, where $a b$ and $c d$ are independent edges in $H, J^{\prime}=H^{\prime}-\{x, y\}$, where $x$ and $y$ are adjacent vertices in $H^{\prime}$, and let $a^{\prime}, b^{\prime}$ and $c^{\prime}, d^{\prime}$ be the other neighbours of $x$ and $y$ in $H^{\prime}$, respectively. Then the dot product $H \cdot H^{\prime}$ is defined as the graph

$$
\left(V(H) \cup V\left(J^{\prime}\right), E(J) \cup E\left(J^{\prime}\right) \cup\left\{a a^{\prime}, b b^{\prime}, c c^{\prime}, d d^{\prime}\right\}\right) .
$$

(Note that under above conditions, the dot product may be disconnected. The dot product was defined by Isaacs in [10]. In fact, following Skupień [14], it seems that this operation was invented earlier by Adel'son-Vel'skiǐ and Titov [1].) 


\section{INFINITELY MANY PLANAR CUBIC HYPOHAMILTONIAN GRAPHS OF GIRTH 53}

Theorem 1. Let $H$ and $H^{\prime}$ be cubic graphs, $H$ non-hamiltonian and $H^{\prime}$ hypohamiltonian. Consider independent edges $a b, c d \in E(H)$. If each of

$$
(a, c),(a, d),(b, c),(b, d), \text { and }((a, b),(c, d))
$$

is good in $H-\{a b, c d\}$, and for each vertex $t \in V(H)$, at least one of $(a, b)$ or $(c, d)$ is good in $H-\{t, a b, c d\}$, then $H \cdot H^{\prime}$ is a cubic hypohamiltonian graph as well. If $H$ and $H^{\prime}$ are planar, and $a b$ and $c d$ lie on the same facial cycle, then the dot product can be applied such that $H \cdot H^{\prime}$ is planar as well. If $g\left(g^{\prime}\right)$ is the girth of $H\left(H^{\prime}\right)$, then the girth of $H \cdot H^{\prime}$ is at least $\min \left\{g, g^{\prime}\right\}$.

Proof. Put $G=H \cdot H^{\prime}$ and denote by $x, y$ the (adjacent) vertices which were deleted from $H^{\prime}$ to form the dot product. $G$ is obviously cubic. Put $N(x)=\left\{a^{\prime}, b^{\prime}, y\right\}$ and $N(y)=\left\{c^{\prime}, d^{\prime}, x\right\}$ such that the unique neighbour of $a^{\prime}\left(b^{\prime}, c^{\prime}, d^{\prime}\right)$ in $H$ is $a(b, c, d)$. We treat $H-\{a b, c d\}=J$ and $H^{\prime}-\{x, y\}=J^{\prime}$ as subgraphs of $G$. W.l.o.g. we may assume that the aforementioned labeling of vertices satisfies that if $H$ and $H^{\prime}$ are planar, then $G$ is also planar.

We first show that $G$ is non-hamiltonian. Assume there exists a hamiltonian cycle $\mathfrak{h}$ in $G$. If $\mathfrak{h} \cap J$ consists of two components $P$ and $P^{\prime}$, then either $\mathfrak{h} \cap J+a b+c d$ is a hamiltonian cycle in $H$, a contradiction since $H$ is non-hamiltonian, or $P$ is a path with end-vertices $a$ and $b$, and $P^{\prime}$ is a path with end-vertices $c$ and $d$. But then $\mathfrak{h} \cap J^{\prime}$ consists of two disjoint paths which together with $a^{\prime} x b^{\prime}$ and $c^{\prime} y d^{\prime}$ form a hamiltonian cycle in $H^{\prime}$, a contradiction since $H^{\prime}$ is hypohamiltonian. So $\mathfrak{h} \cap J$ consists of one component. If $\mathfrak{h} \cap J$ has end-vertices $a, b$ or $c, d$, then we immediately obtain a contradiction (by adding the edge $a b$ or $c d$ ) to the non-hamiltonicity of $H$, so we may assume that $\mathfrak{h} \cap J^{\prime}$ is a hamiltonian path $P$ in $J^{\prime}$ with end-vertices $p \in\left\{a^{\prime}, b^{\prime}\right\}$ and $q \in\left\{c^{\prime}, d^{\prime}\right\}$. Then $P \cup p x y q$ is a hamiltonian cycle in $H^{\prime}$, once again a contradiction.

It remains to show that $G-s$ is hamiltonian for every $s \in V(G)$. Let $s \in V\left(J^{\prime}\right)$. Since $H^{\prime}$ is hypohamiltonian, there exists a hamiltonian cycle $\mathfrak{h}$ in $H^{\prime}-s$. Put $Q=a^{\prime} x b^{\prime}$ and $Q^{\prime}=c^{\prime} y d^{\prime}$. Note that if exactly one of $Q$ and $Q^{\prime}$ lies in $\mathfrak{h}$, then necessarily $s \in\{x, y\}$, which is impossible, as neither $x$ nor $y$ lie in $J^{\prime}$.

Case 1: Neither $Q$ nor $Q^{\prime}$ lie in $\mathfrak{h}$. In this situation, $x y \in E(\mathfrak{h})$, and say $a^{\prime}$ and $d^{\prime}$ are the end-vertices of the path $P^{\prime}=\mathfrak{h}-\{x, y\}$, which is a hamiltonian path in $J^{\prime}-s$. $(a, d)$ is good in $J$, i.e. there exists a hamiltonian path $P$ in $J$ with end-vertices $a, d$, so we obtain the hamiltonian cycle $P^{\prime} \cup P+a a^{\prime}+d d^{\prime}$ in $G-s$ as desired. (If $P^{\prime}$ has end-vertices other than $a^{\prime}, d^{\prime}$, the argument is very similar. Likewise if $s \in\left\{a^{\prime}, b^{\prime}, c^{\prime}, d^{\prime}\right\}$.)

Case 2: Both $Q$ and $Q^{\prime}$ lie in $\mathfrak{h}$. $((a, b),(c, d))$ is good in $H$, so there exist disjoint paths $P_{1}, P_{2}$ in $H$ which together span $H$, with end-vertices $a, b$ and $c, d$, respectively. Denote by $P_{1}^{\prime}, P_{2}^{\prime}$ the components of $\mathfrak{h}-\{x, y\}$. Then $P_{1} \cup P_{2} \cup P_{1}^{\prime} \cup P_{2}^{\prime}+a a^{\prime}+b b^{\prime}+c c^{\prime}+d d^{\prime}$ is a hamiltonian cycle in $G-s$.

Now consider $s \in V(H)$. We know that at least one of $(a, b)$ and $(c, d)$ is good in $H-\{s, a b, c d\}$, say $(a, b)$. So there exists a hamiltonian path $P$ in $H-\{s, a b, c d\}$ with end-vertices $a$ and $b$. Since $H^{\prime}$ is hypohamiltonian, $H^{\prime}-y$ is hamiltonian, which implies that there exists a hamiltonian path $P^{\prime}$ in $J^{\prime}$ with end-vertices $a^{\prime}$ and $b^{\prime}$. Now 


\section{JOURNAL OF GRAPH THEORY}

$P \cup P^{\prime}+a a^{\prime}+b b^{\prime}$ is a hamiltonian cycle in $G-s$ (here it is crucial that $c d \notin E(P)$, as $c d \in E(H)$, but $c d \notin E(G))$. If $(c, d)$ is good in $H-\{s, a b, c d\}$ and not $(a, b)$, we use the fact that $H^{\prime}-x$ is hamiltonian.

Finally, let $g$ be the girth of $H$ and $g^{\prime}$ be the girth of $H^{\prime}$. Assume the girth of $H \cdot H^{\prime}$ is $k<\min \left\{g, g^{\prime}\right\}$, and let $C$ be a cycle of length $k$ in $H \cdot H^{\prime}$. Clearly, $C$ contains (at least) two edges $e_{1}, e_{2} \in\left\{a a^{\prime}, b b^{\prime}, c c^{\prime}, d d^{\prime}\right\}$ with $e_{1}=a a^{\prime}$ w.l.o.g. If $e_{2}=b b^{\prime}$, then $\left(C \cap J^{\prime}\right) \cup a^{\prime} x b^{\prime}$ is a cycle of length at most $k-2$ in $H^{\prime}$. If $e_{2}=c c^{\prime}$, then $\left(C \cap J^{\prime}\right) \cup a^{\prime} x y c^{\prime}$ is a cycle of length at most $k$ in $H^{\prime}$. If $k<g^{\prime}$, in both cases we have a contradiction, so $k \geq g^{\prime}$. But this is impossible, as $k<\min \left\{g, g^{\prime}\right\}$. We have shown that the girth of $H \cdot H^{\prime}$ is at least $\min \left\{g, g^{\prime}\right\}$.

The above result is inspired by a theorem of Fiorini [5], who showed that gluing two hypohamiltonian snarks using the dot product yields a new hypohamiltonian snark. Note that in Fiorini's statement the hypotheses he formulates are too weak to prove the result he advertises; Cavicchioli et al. [3] have identified this omission, but we believe their version is also not entirely correct. A more detailed account of this can be found in [7].

In the following, we will call the pair of edges $a b, c d$ from the statement of Theorem 1 suitable. Let $H$ be the graph from Figure 1. It is one of the three planar cubic hypohamiltonian graphs of girth 5 on 76 vertices found by McKay [12]. Using a computer, we found several suitable pairs of edges $a b, c d$ in this graph. Since we will use double-digit numbers to label the vertices, we shall denote the edge $v w$ by $(v, w)$ for better readability.

Lemma 1. Let $H$ be the graph from Figure 1 . The edge pair $(20,28),(52,62)$ is suitable in $H$.

Proof. It is clear from Figure 1 that the edges $(20,28)$ and $(52,62)$ are on the same facial cycle. (This guarantees that the dot product is planar.)

The pairs $(20,52),(20,62),(28,52)$ and $(28,62)$ are good in $H-\{(20,28),(52,62)\}$ due to the following hamiltonian paths, respectively:

- $52,44,36,26,16,8,3,7,14,22,23,15,24,25,34,41,42,35,43,51,61,71,72$, $62,53,45,37,29,30,38,46,55,54,64,63,73,76,70,60,69,75,74,66,65,56$, $47,57,67,68,59,50,58,49,48,40,33,32,39,31,21,13,6,2,1,4,9,17,27,28$, $19,18,10,5,11,12,20$

- $62,53,45,37,29,30,38,46,55,54,64,63,73,72,71,61,52,44,36,26,35,43$, $51,60,70,76,75,69,59,68,74,66,65,56,47,57,67,58,50,42,41,49,48,39$, $32,23,15,24,33,40,34,25,16,8,3,7,14,22,31,21,13,6,2,1,4,9,17,27,28$, $19,18,10,5,11,12,20$

- $52,44,36,26,16,25,24,15,7,14,13,21,30,38,46,55,54,64,63,73,76,70,60$, $69,75,74,66,65,56,47,57,67,68,59,50,58,49,48,39,31,22,23,32,33,40$, $34,41,42,35,43,51,61,71,72,62,53,45,37,29,20,12,6,2,1,3,8,9,4,10,5$, $11,19,18,17,27,28$

- $62,53,45,37,29,20,12,6,13,14,7,15,23,22,31,21,30,38,46,55,54,64,63$, $73,72,71,70,76,75,69,60,51,61,52,44,43,35,42,50,59,68,74,66,65,56$, 


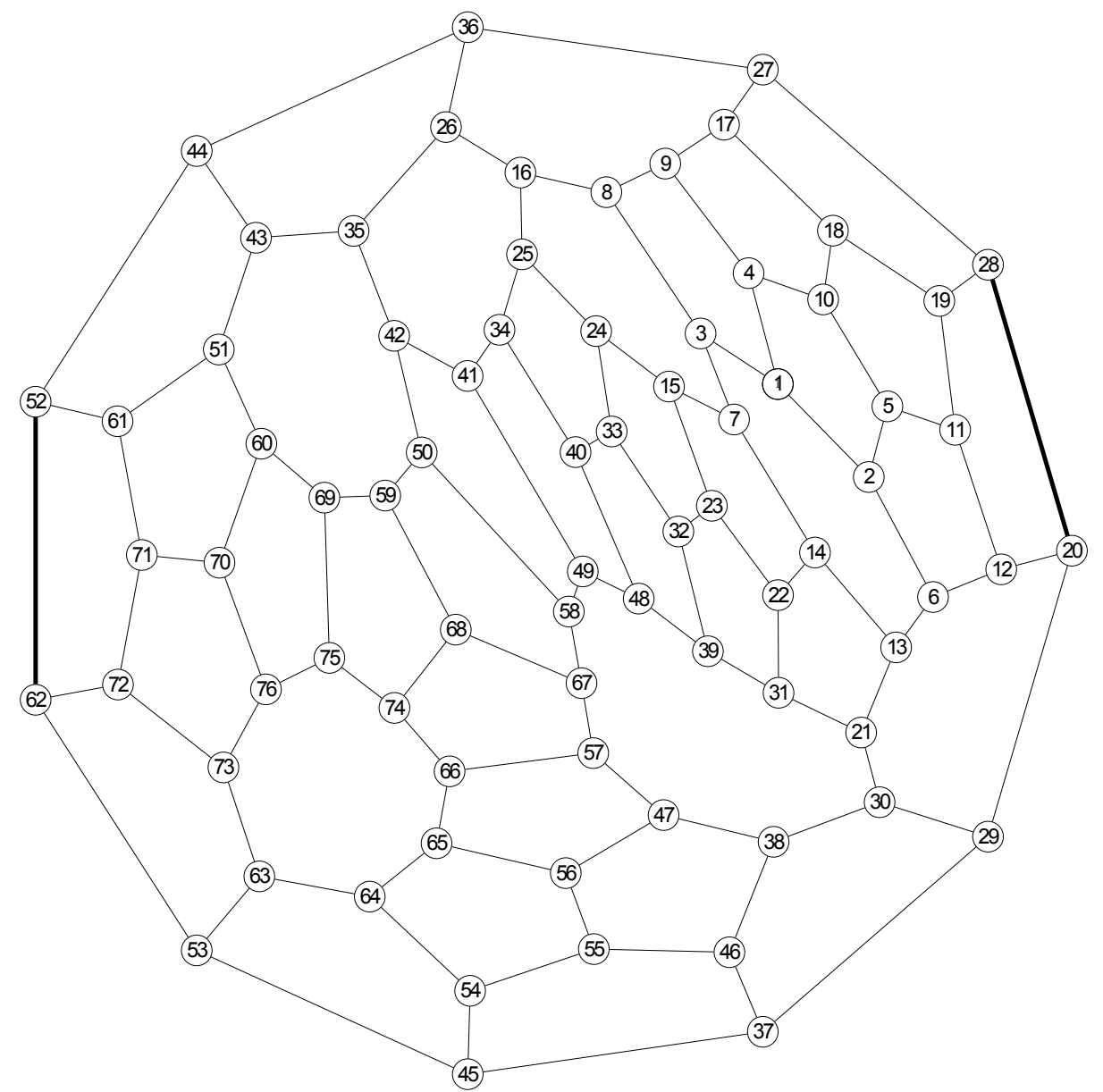

FIGURE 1: The planar cubic hypohamiltonian graph $H$. It has girth 5 and order 76 . $H$ is one of the three smallest planar cubic hypohamiltonian graphs of girth 5 , and was found by McKay [12]. The suitable edge pair $(20,28),(52,62)$ is marked in bold.

$47,57,67,58,49,41,34,40,48,39,32,33,24,25,16,26,36,27,17,18,10,4,9$, $8,3,1,2,5,11,19,28$

Note that $((20,28),(52,62))$ is good in $H-\{(20,28),(52,62)\}$ due to the following two disjoint paths with end-vertices 20 and 28 , and 52 and 62 , respectively, which together span $H$.

- $28,19,11,12,20$ 


\section{JOURNAL OF GRAPH THEORY}

- $62,53,45,37,29,30,21,13,6,2,5,10,18,17,27,36,26,35,42,41,34,25,16$, $8,9,4,1,3,7,14,22,31,39,32,23,15,24,33,40,48,49,58,50,59,69,60,70$, $76,75,74,68,67,57,66,65,56,47,38,46,55,54,64,63,73,72,71,61,51,43$, 44,52

The fact that at least one of $(20,28)$ or $(52,62)$ is good in $H-\{t,(20,28),(52,62)\}$ for every $t \in V(H)$ was shown by computer. We do not include the 76 hamiltonian paths here due to space constraints - 76 paths are necessary since $H$ has trivial automorphism group [12], but in each case we verified that the path found by the computer is indeed a valid hamiltonian path in the graph.

As in Lemma 1, $H$ will denote in the following the 76 -vertex graph from Figure 1. Consider a copy $H^{\prime}$ of $H$. Using the pair of suitable edges lying on the same facial cycle in $H$ we have found in Lemma 1 , and any pair of adjacent vertices in $H^{\prime}$, we form the graph $H \cdot H^{\prime}$. By Theorem $1, H \cdot H^{\prime}$ is a planar cubic hypohamiltonian graph of girth 5. (Theorem 1 states that the girth of $H \cdot H^{\prime}$ is at least 5 , and a planar 3 -connected graph cannot have girth greater than 5.) Iterating this process (i.e., for a copy $H^{\prime \prime}$ of $H$, forming the dot product $H^{\prime \prime} \cdot\left(H \cdot H^{\prime}\right)$, etc.) we obtain the following answer to McKay's question [12]. The second part of the statement was obtained by using the 78-vertex planar cubic hypohamiltonian graph of girth 5 constructed in [6] as $H^{\prime}$ in Theorem 1 .

Theorem 2. There are infinitely many planar cubic hypohamiltonian graphs of girth 5 . More specifically, there exist such graphs of order $n$ for every $n=74 k+a$, where $k \geq 1$ and $a \in\{2,4\}$.

We remark that Theorem 1 may in fact be applied to show that there are smaller graphs which could be used in the above construction, since in its statement we only ask of $H$ to be non-hamiltonian, not hypohamiltonian. However, our focus here was to solve McKay's problem - future work may include a more careful analysis of the graphs which may take the place of $H$.

In 1993, Holton and Sheehan [8] asked if there exists an integer $n_{0}$ such that a planar cubic hypotraceable graph exists for every even integer $\geq n_{0}$. Araya and Wiener [2] settled the question affirmatively with $n_{0}=356$ by applying a method invented by Horton [9] and generalised by Thomassen [16] which combines five hypohamiltonian graphs into a hypotraceable one - in fact, the hypotraceable graphs of Araya and Wiener are not only planar, but 3-connected as well! They also showed that there exists a planar 3-connected cubic hypotraceable graph on 340 vertices. No smaller such graph is known. Applying Thomassen's construction method to the hypohamiltonian graphs described in [18, Theorem 8], we obtain planar 3-connected cubic hypotraceable graphs on $n$ vertices for every even $n \geq 344$, thus improving the $n_{0}$ from the question of Holton and Sheehan.

We end this paper with an application of our result. We recall that before McKay's recent result from [12], all known planar cubic hypohamiltonian graphs had girth 4 . With the above approach - knowing that the hypohamiltonian graphs used contain exactly one 


\section{INFINITELY MANY PLANAR CUBIC HYPOHAMILTONIAN GRAPHS OF GIRTH 57}

quadrilateral face - it is not difficult to verify that the girth of the resulting hypotraceable graph $T$ depends on the selected pair of vertices $x_{i}, y_{i}$ when applying Thomassen's construction method [16]: if for each chosen pair $x_{i}, y_{i}$ at least one of $x_{i}$ or $y_{i}$ lies on the respective quadrilateral face, then $T$ has girth 5 , while if at least one pair is disjoint from the quadrilateral face, then $T$ has girth 4 . In contrast, when applying our Theorem 2 , the choice of any pair $x_{i}, y_{i}$ will yield a planar 3 -connected cubic hypotraceable graph of girth 5 .

\section{References}

[1] G. M. Adel'son-Vel'skiǐ and V. K. Titov. On edge 4-chromatic cubic graphs (in Russian). In Proc. Seminar of 1971 at Moscow Univ., Voprosy Kibernetiki [vol. not numbered], pages 5-14, 1973.

[2] M. Araya and G. Wiener. On cubic planar hypohamiltonian and hypotraceable graphs. Electronic Journal of Combinatorics, 18, \#P85, 2011.

[3] A. Cavicchioli, T. E. Murgolo, B. Ruini, and F. Spaggiari. Special classes of snarks. Acta Applicandae Mathematica, 76(1): 57-88, 2003.

[4] V. Chvátal. Flip-flops in hypohamiltonian graphs. Canadian Mathematical Bulletin, 16(1): 33-41, 1973.

[5] S. Fiorini. Hypohamiltonian snarks. In Graphs and Other Combinatorial Topics (ed.: M. Fiedler), volume 59, pages 70-75. Teubner, 1983.

[6] J. Goedgebeur and C. T. Zamfirescu. Improved bounds for hypohamiltonian graphs. Ars Mathematica Contemporanea, 13(2):235-257, 2017.

[7] J. Goedgebeur and C. T. Zamfirescu. On Hypohamiltonian Snarks and a Theorem of Fiorini. arXiv preprint arXiv:1608.07164. To appear in Ars Mathematica Contemporanea.

[8] D. A. Holton and J. Sheehan. The Petersen Graph, chapter 7: Hypohamiltonian graphs, 1993.

[9] J. D. Horton. A Hypotraceable Graph. Research Report CORR 73-4, Dept. Combin. and Optim., Univ. Waterloo, 1973.

[10] R. Isaacs. Infinite families of nontrivial trivalent graphs which are not Tait colorable. American Mathematical Monthly, 82(3): 221-239, 1975.

[11] M. Jooyandeh, B. D. McKay, P. R. J. Östergård, V. H. Pettersson, and C. T. Zamfirescu. Planar hypohamiltonian graphs on 40 vertices. Journal of Graph Theory, 84(2): 121-133, 2017.

[12] B. D. McKay. Hypohamiltonian planar cubic graphs with girth 5. Journal of Graph Theory, 85(1):7-11, 2017.

[13] K. Ozeki and P. Vrána. 2-edge-Hamiltonian-connectedness of 4-connected plane graphs. European Journal of Combinatorics, 35: 432-448, 2014.

[14] Z. Skupień. Multi-compositions in exponential counting of hypohamiltonian snarks. In Convexity and Discrete Geometry Including Graph Theory, Springer Proc. in Math. \& Stat. (eds.: K. Adiprasito, I. Bárány, C. Vîlcu), volume 148, pages 47-58. Springer, 2016. 


\section{JOURNAL OF GRAPH THEORY}

[15] R. Sousselier. Problème no. 29: Le cercle des irascibles (in French). Revue Française de Recherche Opérationnelle, 7: 405-406, 1963.

[16] C. Thomassen. Planar and infinite hypohamiltonian and hypotraceable graphs. Discrete Mathematics, 14(4): 377-389, 1976.

[17] G. Wiener. Leaf-Critical and Leaf-Stable Graphs. Journal of Graph Theory, 84(4): 443-459, 2017.

[18] C. T. Zamfirescu. On hypohamiltonian and almost hypohamiltonian graphs. Journal of Graph Theory, 79(1):63-81, 2015. 